\title{
Factors Associated with the Symptoms of Young Adults with L5 Spondylolysis
}

\author{
Min-Woo Kim, Kyu Yeol Lee, Seunghyun Lee \\ Department of Orthopedic Surgery, Dong-A University College of Medicine, Busan, Korea
}

\section{Study Design: A retrospective cohort study.}

Purpose: To investigate the factors affecting symptoms in young adults with $L 5$ spondylolysis.

Overview of Literature: L5 spondylolysis is a common disease. However, not all patients diagnosed with L5 spondylolysis exhibit symptoms. This study examined the factors associated with the symptoms of young adults with $\mathrm{L} 5$ spondylolysis.

Methods: The medical records of 70 young adults (mean age, 31.1 years; range, 20-39 years) with $\mathrm{L} 5$ spondylolysis treated at the authors' spine center between March 2008 and February 2015 were reviewed systematically. The symptomatic group ( $n=46$ ) presented with symptoms, such as back pain and/or intermittent lower limb radiating pain, whereas the asymptomatic group ( $\mathrm{n}=24$ ) did not. Age, sex, body mass index (BMI), adjacent disc degeneration, facet degeneration, and measured spino-pelvic parameters (pelvic incidence, sacral slope, pelvic tilt, lumbar lordosis, sacral inclination, and sacral table angle) were investigated with respect to the presence of symptoms. Adjacent disc degeneration was evaluated using T2-weighted sagittal magnetic resonance imaging (MRI, Pfirrmann classification), whereas facet degeneration was evaluated using T2-weighted axial MRI (Grogan classification).

Results: Significant differences in the BMI ( $p=0.032), L 4-5$ disc degeneration $(p=0.030), L 5-S 1$ disc degeneration $(p=0.046), L 4-5$ facet degeneration $(p=0.041)$, and $L 5-S 1$ facet degeneration $(p=0.027)$ were observed between the symptomatic and asymptomatic groups. However, multivariate logistic regression analysis revealed that $L 5-S 1$ disc degeneration $(p=0.033)$ was the only significant factor.

Conclusions: BMI and adjacent disc and facet degeneration may be associated with the manifestation of disease symptoms in young adults with L5 spondylolysis, and the likelihood of the patient exhibiting symptoms increases with increasing severity of L5-S1 disc degeneration.

Keywords: Spondylolysis; Disc; Zygapophyseal joint

\section{Introduction}

Lumbar spondylolysis is a unilateral or bilateral defect of the pars interarticularis [1]. The disease frequently occurs in young adults and most commonly affects the L5 vertebral level, accounting for $85 \%$ of all cases [2]. However, not all patients diagnosed with L5 spondylolysis exhibit symptoms, and the disease may be incidentally diagnosed in asymptomatic individuals during health check-ups. In addition, some individuals with L5 spondylolysis also have disc diseases, multiple-level spondylolysis, spinal stenosis, or another pathological condition of the spine.

Received Jul 6, 2017; Revised Sep 10, 2017; Accepted Oct 15, 2017

Corresponding author: Kyu Yeol Lee

Department of Orthopedic Surgery, Dong-A University College of Medicine, 32 Daesingongwon-ro, Seo-gu, Busan 49201, Korea

Tel: +82-51-240-5166, Fax: +82-51-254-6757, E-mail: gylee@dau.ac.kr 
Therefore, this study examined the factors associated with the symptoms of young adults with L5 spondylolysis. Duval-Beaupère et al. [3] studied many spino-pelvic parameters and reported that the spino-pelvic parameter plays pathologically important roles in various lumbar diseases. Yin et al. [4] compared the spino-pelvic parameters in patients with lumbar spondylolysis and normal adults and concluded that the pelvic incidence (PI) and sacral table angle (STA) are important pathological parameters. Few studies have been conducted to identify the parameters affecting the symptoms of patients with L5 spondylolysis. Therefore, this study examined whether the symptoms exhibited by young adults with L5 spondylolysis are associated with sex, age, body mass index (BMI), sagittal spinopelvic parameters, segmental motion, and adjacent disc and facet degeneration.

\section{Materials and Methods}

We conducted this study in compliance with the principles of the Declaration of Helsinki. The protocol of this study was reviewed and approved by the Institutional Review Board of Dong-A University Hospital (IRB approval no., DAUHIRB-17-011). The informed consent was waived.

Of the patients diagnosed with L5 spondylolysis between March 2008 and February 2015 at the spine center of Dong-A University Hospital, 70 young adults (mean age, 30.9 years; range, $20-39$ years; 50 males and 20 females) were selected for the study. Of these patients, 46 patients had lower back pain or intermittent radiating pain and were classified as the symptomatic group, and 24 patients had no symptoms and were classified as the asymptomatic group. Patients in the asymptomatic group were incidentally diagnosed with spondylolysis at work and school health screenings and were included in the study using magnetic resonance imaging (MRI) voluntarily conducted at our health care center. The demographic and radiographic parameters were analyzed retrospectively. The exclusion criteria were (1) lumbar infection, abscess, or pelvic or hip joint disease; (2) spondylolisthesis; (3) previous lumbar spinal fusion or instrumentation surgery; and (4) presence of spondylolysis at other lumbar levels, such as L3 or L4.

Age, sex, and BMI were measured and compared. The demographic data are shown in Table 1.

Adjacent disc degeneration was examined at L4-5 and L5-S1 using MRI. Disc degeneration at L4-5 and L5-S1 was graded using T2-weighted MRI. The disc structure, extent of distinction between nucleus pulposus and annulus fibrosus, signal intensity of disc degeneration, and height of disc degeneration were examined in the sagittal views. According to the Pfirmann classification system [5], the patients were classified as grades 1-5 (Table 2). Adjacent facet joint degeneration was also examined at L4-5 and L5-S1 using MRI. Cartilage status, osteophytes, and sclerotic change were examined in the axial view. The cases were graded using the Grogan classification system [6]. If the grade of the facet joint degeneration on the left and right was different, the higher grade was selected. The facet degeneration index (FDI) was calculated by adding the three grades of Grogan classification (Table 2). The sides of more degenerated facet joints with the highest FDI were selected for the analysis. The severity of facet joint degeneration was graded according to the highest FDI value.

Adjacent disc and facet joint degeneration in the two study groups were compared, and the association between disc and facet joint degeneration was analyzed using the Pearson correlation coefficient. The groups were subdivided according to age $(20-24,25-29,30-34$, and 35-39 years) to examine the associations between age and the changes in adjacent disc and facet joint degeneration.

When lumbar lateral radiographic images were obtained, the patients were asked to straighten their knees and chest, flex the shoulder joint, and maintain joint flexion to avoid being exposed. Both hip joints were included in the images [7]. In addition, the image was obtained with lumbar flexion and extension on standard lumbar lateral radiography, and the segmental motion of L5-S1 was assessed [8]. The PI, sacral slope (SS), pelvic tilt (PT), lumbar lordosis (LL), sacral inclination (SI), and STA were measured on the lumbar lateral radiographic images. PI was defined as the angle between the line perpendicular to the superior sacral endplate and the line connecting the midpoint of the superior sacral endplate and femoral head axis; SS as the angle between the superior sacral endplate and the horizontal line; PT as the angle between the line connecting the midpoint of the superior sacral endplate to the femoral head and vertical axes; LL as the angle between the superior sacral endplate and superior endplate of L1; SI as the angle between the trailing edge line and the vertical; and STA as the angle between the superior sacral endplate and the trailing edge line of the sacrum. Segmental motion was assessed using lateral 
flexion and extension radiographs of the lumbar spine. Segmental motion at L5-S1 was assessed when spondylolysis was present [9]. Segmental motion at L5-S1, i.e., the angle between the superior endplate of $\mathrm{S} 1$ and the inferior endplate of L5, was measured during lumbar flexion and lumbar extension. The difference between the angles was calculated $[8,10]$.

The mean spino-pelvic parameter measurements and MRI scoring were performed by two spine specialists. The intraclass correlation coefficient (ICC) was measured to determine the reliability of the assessment between the two researchers, and as a result, the ICC value was 0.674 , indicating a sufficient level of the inter-rater reliability.

Continuous and categorical variables were analyzed using the Student $t$-test and chi-square test, respectively. The association between adjacent disc and facet joint degeneration was investigated using Pearson correlation analysis, and the associations between age and adjacent disc and facet joint degeneration were assessed using the Kruskal-Wallis test. Univariate and multivariate logistic regression analyses of the patients' age, sex, BMI, and adjacent disc and facet joint degeneration, which are factors affecting symptoms, were performed. Statistical analysis was performed using IBM SPSS ver. 21.0 (IBM Corp., Armonk, NY, USA), and $p<0.05$ was considered statistically significant.

\section{Results}

The symptomatic group consisted of 31 males (67.4\%) and 15 females (32.6\%), with a mean age of 32.1 years (range, 20-39 years), and the asymptomatic group consisted of 19 males (79.2\%) and five females (20.8\%), with a mean age of 28.8 years. Intergroup differences were observed for age $(p=0.119)$ and sex $(p=0.449)$. The mean BMI in the symptomatic and asymptomatic groups was $29.2 \pm 3.3 \mathrm{~kg} /$

Table 1. Demographic data

\begin{tabular}{lccc} 
Characteristic & Symptomatic & Asymptomatic & $p$-value \\
\hline Age $(\mathrm{yr})$ & $32.1 \pm 5.2$ & $28.8 \pm 5.9$ & 0.119 \\
\hline Sex & $15(32.6)$ & $5(20.8)$ & 0.449 \\
\hline Female & $31(67.4)$ & $19(79.2)$ & \\
\hline $\begin{array}{l}\text { Male } \\
\begin{array}{l}\text { Body mass index } \\
\left(\mathrm{kg} / \mathrm{m}^{2}\right)\end{array}\end{array}$ & $29.2 \pm 3.3$ & $25.3 \pm 2.3$ & 0.032 \\
\hline
\end{tabular}

Values are presented as mean \pm standard deviation or number (\%).

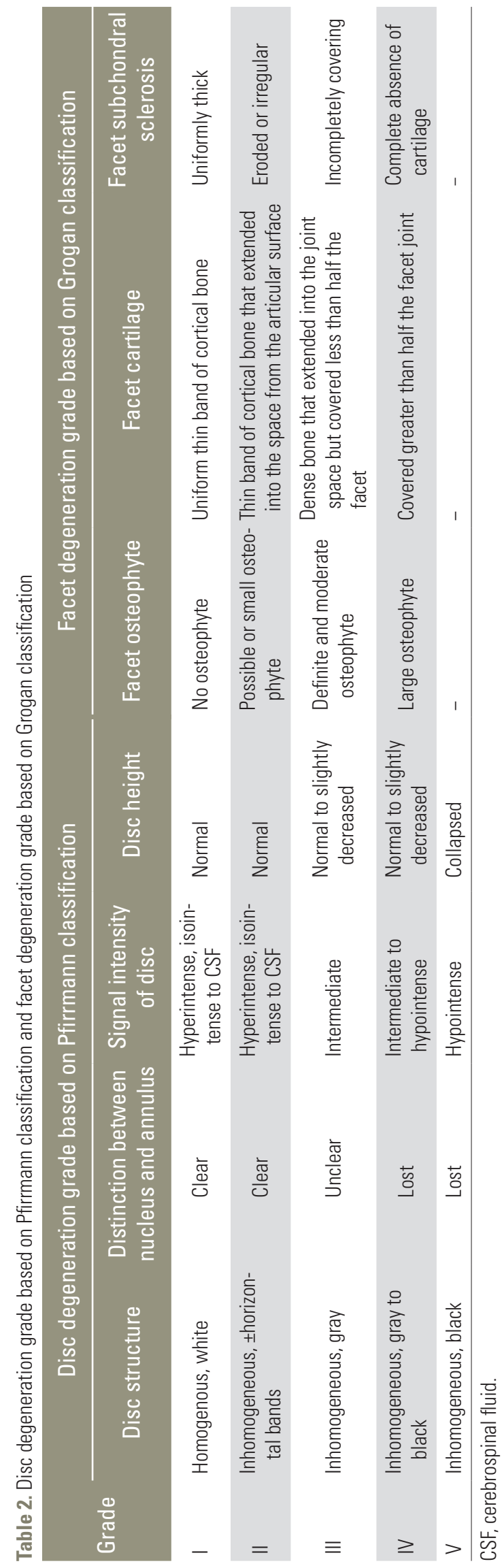


Table 3. Adjacent disc degeneration in symptomatic and asymptomatic groups based on Pfirrmann classification

\begin{tabular}{rccc} 
Variable & $\begin{array}{c}\text { Symptomatic } \\
(\mathrm{n}=46)\end{array}$ & $\begin{array}{c}\text { Asymptomatic } \\
(\mathrm{n}=24)\end{array}$ & $p$-value \\
\hline Disc L4-5 & $2.60 \pm 1.20$ & $1.80 \pm 0.80$ & 0.030 \\
\hline Grade 1 & $7(15.2)$ & $10(41.7)$ & \\
\hline Grade 2 & $18(39.1)$ & $9(37.5)$ & \\
Grade 3 & $15(32.6)$ & $5(20.8)$ & \\
\hline Grade 4 & $3(6.5)$ & 0 & 0.046 \\
\hline Grade 5 & $3(6.5)$ & 0 & \\
\hline Disc L5-S1 & $3.10 \pm 1.10$ & $2.00 \pm 1.00$ & \\
\hline Grade 1 & $2(4.3)$ & $7(29.2)$ & \\
\hline Grade 2 & $12(26.1)$ & $12(50.0)$ & \\
\hline Grade 3 & $15(32.6)$ & $2(8.3)$ & \\
\hline Grade 4 & $12(26.1)$ & $3(12.5)$ & \\
\hline Grade 5 & $5(10.9)$ & 0 & \\
\hline
\end{tabular}

Values are presented as mean \pm standard deviation or number (\%).

$\mathrm{m}^{2}$ and $25.3 \pm 2.3 \mathrm{~kg} / \mathrm{m}^{2}$, respectively. The mean BMI was significantly higher in the symptomatic group than in the asymptomatic group $(p=0.032)$ (Table 1$)$.

Disc degeneration at L4-5 was assessed according to the Pfirrmann classification. In the symptomatic group, 7,18 , 15,0 , and 6 patients were classified as grades $1(15.2 \%), 2$ (39.1\%), 3 (32.6\%), 4 (0\%), and 5 (13.0\%), respectively. In the asymptomatic group, $10,9,5$, and 0 patients were classified as grades 1 (41.7\%), 2 (37.5\%), 3 (20.8\%), and $5(0 \%)$, respectively. Disc degeneration at L5-S1 was also assessed. In the symptomatic group, $2,12,15,12$, and 5 patients were classified as grades 1 (4.3\%), 2 (26.1\%), $3(32.6 \%), 4$ (26.1\%), and $5(10.9 \%)$, respectively. In the asymptomatic group, $7,12,2,3$, and 0 patients were classified as grades 1 (29.2\%), 2 (50\%), 3 (8.3\%), 4 (12.5\%), and $5(0 \%)$, respectively (Table 3 ).

The FDI at L4-5 was assessed according to the Grogan classification. In the symptomatic group, 2, 9, 29, and 6 patients had <3 (4.35\%), 4-6 (19.57\%), 7-9 (63.04\%), and $10-12$ points (13.04\%), respectively. In the asymptomatic group, 3, 18, and 3 patients had $<3$ (12.5\%), 4-6 points (75\%), and $7-9$ points (12.5\%), respectively.

The FDI was also assessed at L5-S1. In the symptomatic group, 1, 7, 27, and 11 patients had $<3$ (2.17\%), 4-6 (15.22\%), 7-9 (57.70\%), and $10-12$ points (23.91\%), respectively. In the asymptomatic group, $2,10,11$, and 1 subjects had <3 (8.33\%), 4-6 (41.67\%), 7-9 (45.83\%), and
Table 4. Adjacent facet joint degeneration in symptomatic and asymptomatic group by facet degeneration index using Grogan classification

\begin{tabular}{cccc} 
Variable & $\begin{array}{c}\text { Symptomatic } \\
(\mathrm{n}=46)\end{array}$ & $\begin{array}{c}\text { Asymptomatic } \\
(\mathrm{n}=24)\end{array}$ & $p$-value \\
\hline Facet L4-5 & $7.58 \pm 3.74$ & $5.66 \pm 2.61$ & 0.041 \\
$\leq 3$ & $2(4.35)$ & $3(12.5)$ & \\
\hline $4-6$ & $9(19.57)$ & $18(75.0)$ & \\
$7-9$ & $29(63.04)$ & $3(12.5)$ & \\
\hline $10-12$ & $6(13.04)$ & 0 & \\
\hline Facet L5-S1 & $8.21 \pm 4.24$ & $6.19 \pm 2.15$ & 0.027 \\
$\leq 3$ & $1(2.17)$ & $2(8.33)$ & \\
\hline $4-6$ & $7(15.22)$ & $10(41.67)$ & \\
\hline $7-9$ & $27(58.70)$ & $11(45.83)$ & \\
\hline $10-12$ & $11(23.91)$ & $1(4.17)$ & \\
\hline
\end{tabular}

Values are presented as mean \pm standard deviation or number (\%).

Table 5. Pearson correlation coefficient between the disc and facet joint

\begin{tabular}{lllll} 
Variable & $\begin{array}{c}\text { Disc } \\
\text { L4-5 }\end{array}$ & $\begin{array}{c}\text { Disc } \\
\text { L5-S1 }\end{array}$ & $\begin{array}{c}\text { Facet } \\
\text { L4-5 }\end{array}$ & $\begin{array}{c}\text { Facet } \\
\text { L5-S1 }\end{array}$ \\
\hline Disc L4-5 & 1.000 & & & \\
Disc L5-S1 & 0.594 & 1.000 & & \\
Facet L4-5 & 0.274 & 0.297 & 1.000 & \\
Facet L5-S1 & 0.108 & 0.176 & 0.587 & 1.000 \\
\hline
\end{tabular}

10-12 points $(4.17 \%)$, respectively (Table 4$)$.

The association between adjacent disc and facet joint degeneration was investigated using the Pearson correlation coefficient. The results were as follows: the Pearson correlation coefficient was 0.594 between L4-5 versus L5-S1 disc degeneration, 0.27 for L4-5 disc degeneration versus L4-5 facet degeneration, 0.108 for L4-5 disc degeneration versus L5-S1 facet degeneration, 0.297 for L5S1 disc degeneration versus L4-5 facet degeneration 0.176 for L5-S1 disc degeneration versus L5-S1 facet degeneration, and 0.587 for L4-5 facet degeneration versus L5-S1 facet degeneration (Table 5).

Significant intergroup differences were observed for adjacent disc degeneration and adjacent facet joint degeneration. The mean grade of disc degeneration at L4-5 was $2.6 \pm 1.2$ and $1.8 \pm 0.8$ in the symptomatic and asymptomatic groups ( $p=0.030)$, respectively; the mean grade of disc degeneration in the two groups at L5-S1 was 3.1 1.1 and $2.0 \pm 1.0$, respectively $(p=0.046)$. The mean FDI at L4-5 


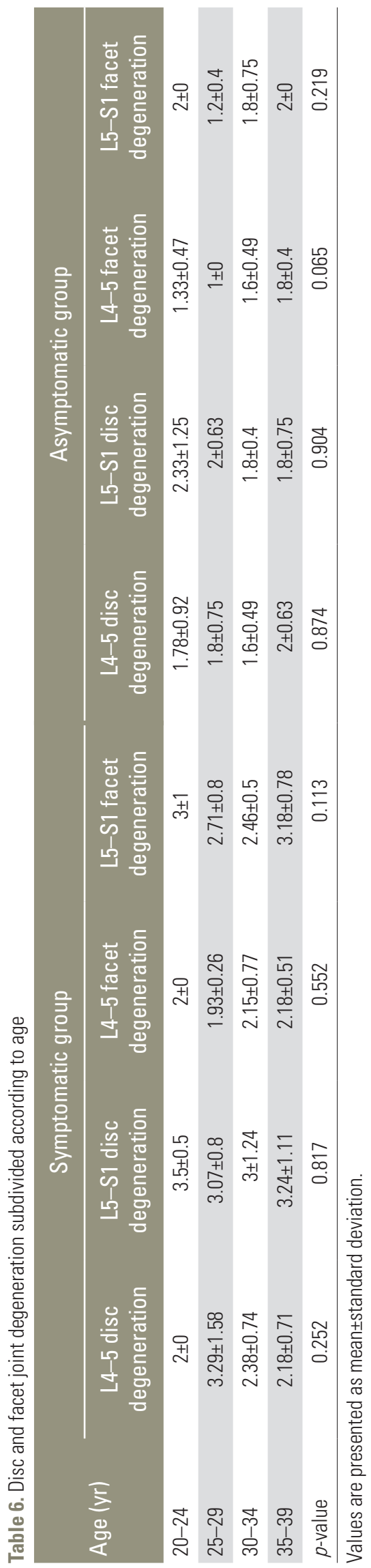

and L5-S1 was 7.58 \pm 3.74 and 5.66 $\pm 2.61(p=0.041)$ and $8.21 \pm 4.24$ and $6.19 \pm 2.15(p=0.027)$ in the symptomatic and asymptomatic groups, respectively (Table 6).

Univariate logistic regression analysis also indicated that the BMI ( $p=0.023), \mathrm{L} 4-5$ disc $(p=0.009), \mathrm{L} 5-\mathrm{S} 1 \mathrm{disc}$ $(p=0.005), \mathrm{L} 4-5$ facet $(p=0.007)$, and L5-S1 facet degeneration ( $p=0.006$ ) were significant factors, but multivariate logistic regression analysis indicated that only L5-S1 disc degeneration ( $p=0.033$ ) had a significant impact (Table 7).

Assessments of lumbar lateral radiographs revealed no significant intergroup differences for six spino-pelvic parameters (PI, PT, SS, SI, LL, and STA) or segmental motion.

The mean PI was $51.0^{\circ} \pm 7.8^{\circ}$ and $44.7^{\circ} \pm 13.8^{\circ}$ in the symptomatic and asymptomatic groups, respectively $(p=0.096)$. The mean SS was $41.7^{\circ} \pm 9.9^{\circ}$ and $33.8^{\circ} \pm 6.0^{\circ}$, respectively $\left(p=0.063\right.$ ); the mean $\mathrm{PT}$ was $16.3^{\circ} \pm 7.6^{\circ}$ and $15.0^{\circ} \pm 12.7^{\circ}$, respectively $(p=0.643)$; the mean SS was $41.7^{\circ} \pm 9.9^{\circ}$ and $33.8^{\circ} \pm 6.0^{\circ}$, respectively $(p=0.063)$; the mean SI was $38.0^{\circ} \pm 10.0^{\circ}$ and $40.8^{\circ} \pm 6.7^{\circ}$, respectively ( $p=0.168$ ); the mean LL was $39.8^{\circ} \pm 14.7^{\circ}$ and $31.6^{\circ} \pm 7.2^{\circ}$, respectively $(p=0.103)$; the mean STA was $94.3^{\circ} \pm 5.6^{\circ}$ and $97.1^{\circ} \pm 6.9^{\circ}$, respectively ( $p=0.092$ ); and the mean segmental motion was $24.0^{\circ} \pm 4.6^{\circ}$ and $21.3^{\circ} \pm 4.2^{\circ}$, respectively (Table 8).

\section{Discussion}

Spondylolysis is defined as a defect in the pars interarticularis of the vertebral arch [1] and is the most common cause of low back pain in children and adolescents [11]. According to the results of a comparative study of adolescent athletes and adults who complain of acute low back pain, spondylolysis occurred in $47 \%$ and $5 \%$ of adolescent athletes and adults, respectively [12]. Although spondylolysis may have many causes, stress fractures caused by repetitive loading are believed to be the most common cause [13]. A high incidence rate of spondylolysis has been reported in many sports, but its incidence is particularly high in sports requiring lumbar hyperextension $[13,14]$. The prevalence of spondylolysis is generally higher in males than in females [15]. Out of 70 patients selected in our study, 50 were males. The symptomatic and asymptomatic groups consisted of 31 and 19 males and 15 and five females, respectively. Patients with spondylolysis experience pain aggravated by hyperextension of the lumbosacral spine and pain radiating to the lower legs. In fact, 
Table 7. Univariate and multivariate Logistic regression analyses based on factors affecting symptoms in patients with L5 spondylolysis

\begin{tabular}{|c|c|c|c|c|}
\hline \multirow{2}{*}{ Variable } & \multicolumn{2}{|c|}{ Univariate } & \multicolumn{2}{|c|}{ Multivariate } \\
\hline & OR $(95 \% \mathrm{Cl})$ & $p$-value & OR $(95 \% \mathrm{Cl})$ & $p$-value \\
\hline Age & $0.874(0.736-1.037)$ & 0.123 & & \\
\hline Sex & $0.544(0.17-1.739)$ & 0.304 & & \\
\hline Body mass index & $1.119(1.015-1.233)$ & 0.023 & 1.096 (0.947-1.267) & 0.218 \\
\hline Disc L4-5 & 2.331 (1.239-4.385) & 0.009 & $0.88(0.37-2.095)$ & 0.772 \\
\hline Disc L5-S1 & $2.936(1.584-5.442)$ & 0.005 & $3.053(1.091-8.542)$ & 0.033 \\
\hline Facet L4-5 & $10.26(3.191-32.981)$ & 0.007 & $1.175(0.227-6.069)$ & 0.848 \\
\hline Facet L5-S1 & 22.477 (3.107-162.607) & 0.006 & 12.671 (1.516-105.934) & 0.079 \\
\hline
\end{tabular}

$\mathrm{OR}$, odds ratio; $\mathrm{Cl}$, confidence interval.

Table 8. Comparison of the spino-pelvic parameters and segmental angular motion of L5-S1 between the symptomatic and asymptomatic groups

\begin{tabular}{lccc} 
Variable & Symptomatic & Asymptomatic & $p$-value \\
\hline Pelvic incidence $\left(^{\circ}\right)$ & $51.0 \pm 7.8$ & $44.7 \pm 13.8$ & 0.096 \\
\hline Pelvic tilt $\left(^{\circ}\right)$ & $16.3 \pm 7.6$ & $15.0 \pm 12.7$ & 0.643 \\
\hline Sacral slope $\left({ }^{\circ}\right)$ & $41.7 \pm 9.9$ & $33.8 \pm 6.0$ & 0.063 \\
Sacral inclination $\left({ }^{\circ}\right)$ & $38.0 \pm 10.0$ & $40.8 \pm 6.7$ & 0.168 \\
\hline Lumbar lordosis $\left({ }^{\circ}\right)$ & $39.8 \pm 14.7$ & $31.6 \pm 7.2$ & 0.103 \\
\hline Sacral table angle $\left({ }^{\circ}\right)$ & $94.3 \pm 5.6$ & $97.1 \pm 6.9$ & 0.092 \\
\hline Segmental motion $\left({ }^{\circ}\right)$ & $24.0 \pm 4.6$ & $21.3 \pm 4.2$ & 0.605 \\
\hline
\end{tabular}

Values are presented as mean \pm standard deviation.

pain is caused by lumbar extension and rotation in $>90 \%$ of patients with spondylolysis [16]. On physical examination, severe local tenderness, particularly in the lower back, determined by the stork or hyperextension test is observed. Muscle spasms are present around the spine, and tight hamstrings are found in approximately $70 \%$ of patients. However, radiating pain is rare [17]. Spondylolysis rarely has a noticeable deformity, but lumbar hyperlordosis and flat buttocks are observed in spondylolisthesis. Spondylolysis can be diagnosed by the "Scottie dog" sign.

A pars defect or fracture of L5 may alter the biomechanics of the spine, particularly at the L4-5 and L5S1 segments, and may increase the stress and strain on the discs and facet joints of L4-5 and L5-S1 during axial loading or body motion. Adjacent disc degeneration was speculated to be strongly associated with pain in patients with L5 spondylolysis. In the present study, L4-5 ( $p=0.030)$ and L5-S1 disc degeneration $(p=0.046)$ were significantly associated with the presence of symptoms. Adjacent facet joint degeneration was also expected to affect the symptoms, and our results revealed that L4-5 facet joint de- generation ( $p=0.041)$ and L5-S1 facet joint degeneration ( $p=0.027$ ) were significantly associated with the presence of symptoms. Accordingly, disc and facet joint degeneration could be significant factors affecting the symptoms observed in patients with L5 spondylolysis. These results suggested that the degenerative changes in adjacent discs and facet joints significantly contribute to the symptoms in patients with L5 spondylolysis (Tables 3, 4).

In the present study, Pearson correlation coefficients were used to examine the correlations between the two variables. The correlation coefficients for L4-5 disc degeneration versus L5-S1 disc degeneration, L4-5 disc degeneration versus facet joint degeneration, L5-S1 disc degeneration versus L4-5 facet joint degeneration, and L5-S1 disc degeneration versus facet joint degeneration were $0.594,0.274,0.297$, and 0.587 , respectively (Table 5). Overall, these findings indicate that $\mathrm{L} 4-5$ disc degeneration, L5-S1 disc degeneration, L4-5 facet joint degeneration, and L5-S1 facet joint degeneration are correlated with each other.

The cohort was divided into four sub-groups according 
to age (20-24, 25-29, 30-34, and 35-39 years) to examine the associations between patient age and adjacent disc and facet joint degeneration. The mean grade in the symptomatic group was greater than that in the asymptomatic group. In the symptomatic and asymptomatic groups, adjacent disc and facet joint degeneration did not increase significantly with age ( $p=0.252$ and 0.219 , respectively). Furthermore, age-related degeneration was not significant for those aged 20-39 years with L5 spondylolysis (Table 6).

Spondylolysis can also be a risk factor for disc and facet joint degeneration. Therefore, univariate and multivariate logistic regression analyses of the patients' age, sex, BMI, and adjacent disc and facet joint degeneration, which are factors affecting the symptoms, were performed. Univariate logistic regression analysis revealed that $\mathrm{L} 4-5$ disc, L4-5 facet, L5-S1 disc, and L5-S1 facet degeneration were significant factors, whereas multivariate logistic regression analysis revealed that only L5-S1 disc degeneration had a significant impact. If the odds ratio was $>1$, the likelihood of symptoms increased with increasing value of the independent variable. Therefore, symptoms are more likely to appear with more severe L5-S1 disc degeneration (Table 7).

Recently, Oh et al. [18] reported that the PI and SS in patients with L5 spondylolysis were higher than those in normal subjects, but the STA was lower in patients with L5 spondylolysis. Yin et al. [4] concluded that the PI in patients with spondylolysis was significantly higher than that in normal subjects $(p<0.001)$, and the STA was significantly lower in patients with spondylolysis $(p<0.001)$. Furthermore, the PT, SS, and LL in patients were significantly higher than those in normal subjects $(p<0.05)$. Previous studies reported that the gravity loads on the spine are composed of two components at L5-S1, i.e., a forward shear force along the superior endplate of S1 and a downward compression load perpendicular to the superior endplate of S1. Moreover, the shear force increases gradually from L1 to L5 to a peak at L5. The higher the SS and LL are, the greater the shear force and stress on the L5 pars interarticularis. In contrast, a small STA leads to a greater shear force in the lumbar spine, particularly in the lower lumbar spine, which causes more stress on the pars interarticularis and may result in spondylolysis. In the present study, however, patients with L5 spondylolysis were recruited and then allocated to dedicated groups based on the presence of symptoms. In the two aforementioned studies, significant differences for the SS, PI, and STA were observed between patients and normal controls
$[4,18]$; however, in the present study, no significant intergroup differences were observed for the SS ( $p=0.063$ ), PI $(p=0.096)$, and STA $(p=0.092)$, although these three parameters tended to increase or decrease in the two aforementioned studies. In contrast, no intergroup difference was observed for the other spino-pelvic parameters. The aforementioned two studies focused on spino-pelvic parameters, such as PI, but the results obtained could not to be used to determine if these parameters were responsible for symptoms in patients. However, these results did not suggest that these factors significantly contributed to the symptoms in patients with L5 spondylolysis. Additional large-scale studies will be required to clarify this issue.

This study has several limitations. The type of work, daily lifestyle, and psychosocial factors may contribute to the symptoms of L5 spondylolysis. In particular, spondylolysis occurs more frequently in individuals with jobs that place stress on the lower back, such as those involved with carrying heavy objects, standing for long periods, or using vibrating equipment. Furthermore, this study does not provide the biomechanical issues of back pain. Second, data were insufficient to support the results suggesting that BMI contributed to the symptoms in patients with spondylolysis. Third, when patients with spondylolysis were diagnosed, MRI was only rarely performed at our institution. In fact, MRI of the 70 patients was performed at other hospitals or when patients complained of severe pain, suggesting that the patients included in the present study experienced more severe pain than that experienced by the average patient with L5 spondylolysis. Fourth, this was a retrospective single-center study, and the collection of data for the asymptomatic group was limited. This is because generally, MRI was rarely used for asymptomatic patients. For this reason, expanding the subjects further is difficult.

\section{Conclusions}

The present study suggested that BMI and adjacent disc and facet degeneration is associated with the manifestation of disease symptoms in young adults with L5 spondylolysis and that patients with more severe L5-S1 disc degeneration are more likely to exhibit symptoms.

\section{Conflict of Interest}

No potential conflict of interest relevant to this article was reported. 


\section{References}

1. Leone A, Cianfoni A, Cerase A, Magarelli N, Bonomo L. Lumbar spondylolysis: a review. Skeletal Radiol 2011;40:683-700.

2. Hu SS, Tribus CB, Diab M, Ghanayem AJ. Spondylolisthesis and spondylolysis. Instr Course Lect 2008;57:431-45.

3. Duval-Beaupere G, Schmidt C, Cosson P. A Barycentremetric study of the sagittal shape of spine and pelvis: the conditions required for an economic standing position. Ann Biomed Eng 1992;20:451-62.

4. Yin J, Peng BG, Li YC, Zhang NY, Yang L, Li DM. Differences of sagittal lumbosacral parameters between patients with lumbar spondylolysis and normal adults. Chin Med J (Engl) 2016;129:1166-70.

5. Pfirrmann CW, Metzdorf A, Zanetti M, Hodler J, Boos N. Magnetic resonance classification of lumbar intervertebral disc degeneration. Spine (Phila $\mathrm{Pa}$ 1976) 2001;26:1873-8.

6. Grogan J, Nowicki BH, Schmidt TA, Haughton VM. Lumbar facet joint tropism does not accelerate degeneration of the facet joints. AJNR Am J Neuroradiol 1997; 18:1325-9.

7. Horton WC, Brown CW, Bridwell KH, Glassman SD, Suk SI, Cha CW. Is there an optimal patient stance for obtaining a lateral 36 " radiograph?: a critical comparison of three techniques. Spine (Phila Pa 1976) 2005;30:427-33.

8. Frobin W, Brinckmann P, Leivseth G, Biggemann M, Reikeras O. Precision measurement of segmental motion from flexion-extension radiographs of the lumbar spine. Clin Biomech (Bristol, Avon) 1996;11:45765.

9. Klineberg E, Schwab F, Smith JS, Gupta MC, Lafage V, Bess S. Sagittal spinal pelvic alignment. Neurosurg Clin N Am 2013;24:157-62.
10. Boden SD, Wiesel SW. Lumbosacral segmental motion in normal individuals: have we been measuring instability properly? Spine (Phila Pa 1976) 1990;15:571-6.

11. Jones GT, Macfarlane GJ. Epidemiology of low back pain in children and adolescents. Arch Dis Child 2005;90:312-6.

12. Bono CM. Low-back pain in athletes. J Bone Joint Surg Am 2004;86-A:382-96.

13. Soler T, Calderon C. The prevalence of spondylolysis in the Spanish elite athlete. Am J Sports Med 2000;28:57-62.

14. Rosenberg NJ, Bargar WL, Friedman B. The incidence of spondylolysis and spondylolisthesis in nonambulatory patients. Spine (Phila Pa 1976) 1981;6:358.

15. Kalichman L, Kim DH, Li L, Guermazi A, Berkin V, Hunter DJ. Spondylolysis and spondylolisthesis: prevalence and association with low back pain in the adult community-based population. Spine (Phila $\mathrm{Pa}$ 1976) 2009;34:199-205.

16. Blanda J, Bethem D, Moats W, Lew M. Defects of pars interarticularis in athletes: a protocol for nonoperative treatment. J Spinal Disord 1993;6:406-11.

17. Jinkins JR, Matthes JC, Sener RN, Venkatappan S, Rauch R. Spondylolysis, spondylolisthesis, and associated nerve root entrapment in the lumbosacral spine: MR evaluation. AJR Am J Roentgenol 1992;159:799-803.

18. Oh YM, Choi HY, Eun JP. The comparison of sagittal spinopelvic parameters between young adult patients with L5 spondylolysis and age-matched control group. J Korean Neurosurg Soc 2013;54:207-10. 